Musique et Sciences Sociales

$6 \mid 2016$

Lignes d'écoute, écoute en ligne

\title{
Les Lumières à Manhattan
}

ou « cela devait arriver »

Lydia Goehr

\section{(2) OpenEdition}

Journals

\section{Édition électronique}

URL : http://journals.openedition.org/transposition/1649

DOI : $10.4000 /$ transposition. 1649

ISSN : 2110-6134

Éditeur

CRAL - Centre de recherche sur les arts et le langage

\section{Référence électronique}

Lydia Goehr, "Les Lumières à Manhattan », Transposition [En ligne], 6 | 2016, mis en ligne le 20 mars

2017, consulté le 31 juillet 2019. URL : http://journals.openedition.org/transposition/1649 ; DOI :

10.4000/transposition.1649

Ce document a été généré automatiquement le 31 juillet 2019.

\section{(c) (i) (?)}

La revue Transposition est mise à disposition selon les termes de la Licence Creative Commons Attribution - Partage dans les Mêmes Conditions 4.0 International. 


\title{
Les Lumières à Manhattan
}

\author{
ou « cela devait arriver »
}

\author{
Lydia Goehr
}

\section{NOTE DE L'ÉDITEUR}

Ce texte est issu d'une conférence donnée à la Philharmonie de Paris le 21 avril 2015 dans le cadre du week-end Sketches of New York.

1 La Dialectik der Aufklärung in Amerika n'est pas la même chose que la Dialectique des Lumières aux États-Unis. La différence tient sans doute au fait qu'on regarde les choses de l'extérieur ou de l'intérieur du pays. Le titre de cette conférence est volontairement ambigu. Il nous invite à considérer la réception aux États-Unis du livre d'Horkheimer et Adorno, Die Dialektik der Aufklärung ${ }^{1}$, et son rôle dans la définition du concept d' "Amerika » avec un $k$ à la Kafka. Et à nous demander s'il a vraiment existé aux États-Unis, conformément à un idéal "américain ", un concept des Lumières et, dans l'affirmative, si le développement dialectique de ce concept a été suffisant pour unir les différents États du pays. Se pose enfin le problème de l'impact de la dialectique des Lumières, comme livre et comme sujet, dans les pays qui forment le sud plutôt que le nord de l'Amérique.

Dans mon livre Elective Affinities ${ }^{2}$, je suis un des fils rouges des Affinités électives de Goethe pour explorer les relations tendues entre l'Allemagne et l'Amérique à l'époque des Lumières - dont celle concernant l'idée d'opéra américain. J'ai très vite appris que c'était une chose d'écrire une histoire de l'opéra aux États-Unis et que c'en était une autre d'écrire une histoire du concept (Begriff) d'opéra américain. Si la première peut se faire à partir d'une synthèse des traditions de l'opéra, de l'opérette et du music-hall nées de communautés de souche ou immigrées, comme on en trouve partout dans le monde où il y a de l'opéra, l'histoire conceptuelle suppose de montrer si, quand et où l'opéra s'est ancré dans la conscience d'une nation. L'opéra américain en tant que concept n'est pas moins tendu que l'opéra italien, l'opéra français ou l'opéra allemand, mais ces tensions sont très variables selon l'endroit où la scène opératique s'est affirmée comme la représentante de la nation. J'ai découvert que placer l'opéra sous la rubrique de l'identité 
nationale pouvait se passer chez nous comme à l'étranger. En un sens, nous n'avons pas besoin d'un concept d'opéra italien, à moins que cela ne devienne un enjeu pour la nation ou que l'opéra d'Italie commence à voyager et à dominer la vie musicale de pays étrangers. Je me suis aussi rendu compte que le concept d'opéra américain était plus compliqué, moins en raison de l'opéra qu'à cause de l'Amérique, étant donné que, malgré la très longue histoire du continent américain, l'« Amérique » a aussi représenté, depuis la lointaine Europe, un idéal de nation. Pendant les décennies de forte immigration européenne, et notamment allemande, aux États-Unis, cet idéal d'«Amérique » et de certains opéras " américains » a traversé l'Atlantique. Mais ce qui est arrivé avec eux dans leurs bagages est souvent entré en conflit avec ce qui était déjà l'opéra de style américain ou états-unien aux USA.

Je me suis longtemps intéressée à la transmission des concepts de part et d'autre de l'Atlantique, et notamment des concepts touchant à l'art de la musique. J'en parle ici aujourd'hui pour vous rappeler la différence entre les histoires empiriques conceptuelles qui font et défont les nations. Il en va du concept d'opéra comme du concept des Lumières : c'est une chose de retracer le mouvement des Lumières aux États-Unis, c'en est une autre de se demander ce que l'« Amérique » représente à l'intérieur et à l'extérieur de la nation selon que le concept suit un mouvement dialectique (Bewegung) ou un autoenveloppement (sich-Entfalten). Pour Hegel et pour beaucoup d'Européens du début du XIX ${ }^{e}$ siècle, l'Amérique était considérée comme une possibilité utopique; elle représentait une promesse de bonheur et de liberté, tandis que la raison et le progrès se frayaient un chemin laborieux et douloureux sur le continent européen. Cependant, en même temps, un schéma dialectique comparable était à l'œuvre aux États-Unis : il y avait une histoire de l'unification difficile des États, et il y avait un idéal de ce que l'« Amérique » était censée représenter.

4 Suivre le dialektische Bewegung du concept d'Aufklärung, c'est observer le mouvement de points de vue opposés sur la réalité et l'idéalité qui sont associées à l'avènement et à la disparition des nations. On le voit bien dans le livre d'Horkheimer et Adorno quand on examine les diverses références à l'«Amérique ", à l'«Allemagne» et à l'« Europe ». Depuis l'Allemagne, en effet, ils voyaient dans l'Amérique la promesse d'une résistance au fascisme et au nazisme, tandis que depuis les États-Unis, ils voyaient dans une Allemagne vaincue et en ruines les traces archaïques d'une résistance à un capitalisme devenu en Amérique, dans l'administration et l'industrialisation quasi-totale de ses formes sociales et culturelles, extrême. Les références à l'« Allemagne » et à l'« Amérique » étaient aussi chargées dialectiquement que l'emploi du mot "catastrophe»ou, dans des écrits ultérieurs, celui du mot « Auschwitz », puisque ces mots représentaient des constellations de tendances sociales - Tendenzen. Dans leur livre, Horkheimer et Adorno font des allersretours entre l'Allemagne/Europe et l'Amérique afin de retracer non seulement le progrès du capitalisme contre la démocratie et l'égalité, mais aussi celui de la démocratisation contre la démocratie et les Lumières. Si l'idéal d'« Amerika » a produit des frictions compte tenu de la réalité des États-Unis, alors l'idée d'« Allemagne » s'est elle aussi scindée en deux, entre ce qu'elle avait produit au moyen d'une rationalisation avancée et ce que Thomas Mann, par exemple, emportait avec lui quand il disait que l'Allemagne telle qu'elle aurait dû être, mais comme elle n'était pas, le suivait partout en exil.

Permettez-moi de vous rappeler mon anecdote favorite, celle de deux écrivains allemands, assis dans un petit bateau, au milieu de l'Atlantique: Thomas Mann et Erich 
Kahler. La Deuxième Guerre mondiale arrive à sa fin et tout le monde sait qui a gagné et perdu la guerre - ou du moins tout le monde croit le savoir. Un des deux écrivains part aux États-Unis après avoir beaucoup souffert en Allemagne (en réalité, Kahler y est parti bien avant); l'autre rentre en Allemagne après avoir souffert d'une autre manière dans son exil ensoleillé sur une palissade qui, comme le mot l'indique, permettait de délimiter le quartier que l'on a appelé "Weimar sur le Pacifique» (Weimar on the Pacific). Passant sur une vague vertigineuse, chacun posait à l'autre cette question rhétorique: «Es-tu fou? » De nos jours, il est vrai, la folie n'est pas aussi évidente que la folie évoquée dans le livre d'Horkheimer et Adorno en 1944. Mais elle est bien visible dans l'onde de choc du tableau quelque peu extrême qu'ils y dessinent tous deux.

6 Dans mon travail, je me suis souvent retrouvée naufragée, non pas dans une barque, mais au milieu des airs, quelque part au-dessus de l'Atlantique, me demandant dans quelle direction diriger mon regard : vers l'Europe ou vers les États-Unis ? Et j'ai souvent été arrêtée en chemin au tout début des choses: quel titre retenir, comment rédiger la première phrase, quels exemples utiliser à l'appui d'un schéma de pensée ? Pour cette conférence, ce n'est pas seulement le titre qui m'a arrêtée, mais aussi l'image devant lui servir de publicité.

7 Cette image, je me permets de le rappeler, n'a rien de monumental dans ses dimensions ; c'est une chromolithographie mesurant 30,48 sur 40,64 cm. Mais c'est un monument d'inspiration. Intitulée "Progrès américain » (American Progress), elle a été réalisée en 1872 par l'artiste américain John Gast pour servir d'allégorie à ce qu'on appelle aux ÉtatsUnis et à propos des États-Unis la "destinée manifeste " (Manifest Destiny). Une figure suspendue, schwebenden, nommée Columbia, plane au-dessus d'un paysage agreste Columbia est d'ailleurs le nom de l'université où j'enseigne depuis vingt ans. Telle université, telle image : Columbia apporte la lumière aux colons qui traversent le pays d'est en ouest. Le mouvement vers l'ouest du progrès peut être interprété à la fois comme la continuation du mouvement de l'Europe à l'Amérique, et comme le signe d'une rupture et d'une conversion. Avec une sanction divine, le mouvement vers l'ouest a commencé sur la côte est, en rupture avec les mouvements comparables en Europe. Columbia lance le mouvement d'un Nouveau Monde séparé d'un Vieux Monde, dont le propre mouvement, par épuisement, par Europamüde, est arrivé à un terme sans même avoir réalisé la fin envisagée.

8 C'est en tout cas comme cela que Frederick Merk, historien de la guerre de Sécession et de l'esclavage, professeur à Harvard, interprète cette image. Il y voit « les potentialités d'une terre nouvelle où un nouvel éden pourra être bâti ${ }^{3}$ " sur Terre. Dans son livre de 1963 intitulé Manifest Destiny and Mission in American History, il voit dans les Lumières une nécessité et une norme d'expansionnisme, au sens de processus de correction permanent des erreurs du passé, à la fois dans le Vieux Monde qu'est l'Europe et dans l'histoire des États du Nouveau Monde, qui sont en voie de lente unification. Dans sa préface, il nous avertit que, bien qu'elle soit passionnante, cette histoire de l'expansionnisme des ÉtatsUnis ne devrait pas "enthousiasmer ${ }^{4}$ " ses lecteurs, compte tenu de l'oppression inévitable - et, pour certains, justifiée - impliquée par ladite expansion. Car l'expansionnisme est - et ne peut qu'être, si l'on en croit certains - «le rude coude à coude des propriétaires d'une terre, d'un côté, et le processus de leur expropriation, de l'autre ${ }^{5}$ ». Dans cette phrase, les "propriétaires » sont par hypothèse tous ceux qui ont acquis, presque par nature, une propriété foncière, et qui se sont heurtés aux 
spéculateurs aux coudes puissants pour qui le capital n'est qu'un moyen de tout s'approprier.

9 Merk parle de son désir d'écrire l'histoire tumultueuse de la "destinée manifeste » en suivant un principe emprunté au philosophe espagnol George Santayana : «Qui ne peut se souvenir du passé est condamné à le répéter ${ }^{6}$. (Cette phrase sonne pour nous aujourd'hui comme un slogan de la philosophie de l'histoire. Elle est issue de The Life of Reason, une œuvre en cinq tomes dans laquelle il aborde les dangers de l'immuabilité des croyances et des sentiments, et en particulier le risque de trop faire confiance à ce qui est censé aller de soi ou relever du sens commun ${ }^{7}$. Il compare l'écriture de l'histoire à celle d'une vie humaine. Car dans l'une comme dans l'autre, il ne peut y avoir progrès que si l'on n'oublie pas ce que l'expérience nous apprend, à condition toutefois qu'apprendre de l'expérience signifie se soumettre encore et encore à l'expérience brute de la réflexion, notamment toutes les fois que la vérité ou la signification de l'expérience paraissent le plus évidents et le plus sensés. Comme l'ont montré Collingwood, John Dewey et de nombreux autres pragmatistes après lui, on tire généralement une pensée de l'expérience pour pouvoir en faire un usage nouveau, même si, en extrapolant cette pensée, il faut prendre soin de l'adapter. Car une pensée qui commence bien, de laquelle on peut apprendre, peut être trahie si elle est adaptée à de mauvais usages, tout comme, inversement, il est possible de faire un bon usage d'une pensée qui commence mal, mais de laquelle on peut aussi apprendre. Ne soyez pas surpris que tout cela fasse songer à la vision nietzschéenne de l'utilité et de l'inconvénient pour la vie non pas de l'histoire, mais des concepts...

Mais revenons à notre image. La figure de Columbia, dans l'esprit de la destinée manifeste et de l'expansionnisme, encourage les pionniers à "repousser", " coloniser» ou «domestiquer» toujours plus à l'ouest l'histoire naturelle et agraire des populations indiennes et des animaux. Le mouvement correspond à une vision téléologique du progrès, souvent légitimée par une croyance dans la divine providence. Les gens se déplacent sur la terre, guidés par un idéal. Columbia tient dans ses mains l'avenir de la technologie, un fil de télégraphe, et celui de l'éducation, un livre d'école. Technologie + éducation = progrès. Derrière l'idée de destinée manifeste, mais coupée de cette image, il y a l'idée de ce que le géographe allemand Friedrich Ratzel, en 1901, a finalement baptisé le Lebensraum ${ }^{8}$. Quelque vingt ans plus tard, cette idée a été pervertie au nom de la cause aryenne. Mais cela ne fait que rendre manifeste qu'il y a ici une mince frontière, trop mince parfois, entre le bon et le mauvais usage, entre un progrès allant croissant vers un éclairement et un progrès régressant vers la catastrophe.

11 Sur cette mince frontière, j'imagine qu'Adorno aurait eu du mal à tenir, qu'il aurait regardé ce qu'était devenue la destinée manifeste aux États-Unis et qu'il se serait demandé si son idée de 1932 - composer un opéra moderniste ou dissonant sur le trésor ( der Schatz) de Joe l'Indien - aurait vraiment permis de révéler les dangers de l'aventure américaine contemporaine. Là encore, nous n'avons pas besoin d'Adorno pour nous interroger nous aussi, car l'idée de "destinée manifeste» pour les États-Unis a été contestée dès le début, avant même que la formule ne fut introduite dans le discours. Et par cette contestation, les critiques allaient déjà en ville pour montrer que l'entreprise capitaliste - Unternehmen - se débarrassait de toutes les dimensions éthiques de ce que signifiait pour les Américains « se lancer dans l'aventure »-Abenteuer.

12 Dans les années 1770 , l'idée d'Amérique était considérée comme une cité nouvelle - une nouvelle Jérusalem - que l'on bâtirait au sommet d'une colline. C'était une vision de lumières, d'émancipation et même d'exode par rapport au pouvoir étranger anglais, 
même s'il était permis d'emporter en terre promise ce qu'on avait appris dans le vieux pays. À la cité sur la colline, on ne cessait de demander si ce qui apparaissait dans la loi et dans la constitution était fidèle à ce que semblait exiger l'idéal. L'égalité et la liberté étaient-elles compatibles avec la colonisation, l'esclavage, le capital et la conquête ? L'idée américaine de destinée manifeste était-elle compatible avec ce qu'exigeait la gouvernance des États-Unis?

Quand la formule de la « destinée manifeste » a été introduite, en 1845, par le directeur de journal et démocrate américain John O'Sullivan, l'idée était déjà bancale. Comme si, dans l'image, Columbia flottait trop haut et était trop libre - ou même hostile - pour les pionniers emportés dans leur mouvement vers l'ouest. Sullivan a glissé la formule dans un article du New York Morning News pour critiquer l'expansion territoriale vers la lointaine côte ouest. Et, bien que sa destinée manifeste fût déjà bancale, on se l'est immédiatement appropriée pour réaffirmer ce que les Puritains avaient emporté à l'origine en Amérique sur un bateau : une croyance dans l'expansionnisme, justifiée par la conviction que l'Amérique était exceptionnelle. En 1778, dans un texte intitulé Common Sense, et traduit en français en 1791 sous le titre le Sens commun, un certain Thomas Paine disait des citoyens des futurs États-Unis d'Amérique : «Il ne tient qu'à nous de revenir aux premiers âges du monde. On n'a pas vu, depuis Noé, de peuple dans une pareille situation ${ }^{9}$.» Cette sorte d'exceptionnalisme a été reprise partout et dans de nombreux discours prononcés au nom du pays : dans la Déclaration d'indépendance, dans l'adresse de Gettysburg, dans l'hymne «America the Beautiful » où l'on chante «from sea to shining sea» (de la mer à la mer étincelante), et même dans le rêve de Martin Luther King d'un pays où chacun serait égal devant la loi. Or, il fallait, pour que l'exceptionnalisme puisse soutenir l'expansionnisme, qu'il devienne la vision communément admise d'une nouvelle communauté nationale, c'est-à-dire qu'il soit évident pour tous.

En 1861, l'Allemand Emanuel Leutze réalisait un grand tableau intitulé Westward the Course of Empire Takes Its Way (L'empire s'étend toujours plus à l'ouest). Il montre des migrants venant de l'est et s'acheminant vers les hauteurs de l'ouest, d'où l'on aperçoit une terre bénie et lumineuse. Sortant de l'obscurité, on y voit des personnages couverts de bandages et de cicatrices, témoignages de leurs luttes et de leurs souffrances. On comprend qu'ils ont déjà fait un long voyage ; ils entrevoient déjà le but que désigne le pèlerin, très inspiré par des figures bibliques et historiques : une madone, Moïse et les rois mages, Christophe Colomb, etc. Leutze tirait son titre d'un poème de 1826, écrit avec un zèle missionnaire par un philosophe bien connu :

La Muse, dégoûtée par un âge et des cieux

Où ne demeure plus un seul sujet glorieux,

Attend des jours meilleurs dans des terres lointaines,

D'où naîtront des sujets enfin dignes de gloire ;

Sous ces cieux bienheureux, où un soleil cordial

Et une terre vierge produisent de telles choses,

La puissance de l'art succombe à la nature,

Et les beautés trompeuses cèdent à la vérité ;

Sous ces cieux bienheureux, où siège l'innocence,

La nature est le guide, la vertu est la reine,

Et l'homme n'impose pas comme sens et vérité

Le pédantisme odieux des juges et des écoles.

Là-bas sera chanté un nouvel âge d'or,

Un empire naîtra, et renaîtront les arts,

La grande et bonne rage épique et inspirée,

Les têtes les plus sages et les cœurs les plus nobles. 
Non comme ceux qu'enfante une Europe épuisée,

Mais de ceux qu'elle offrait dans sa belle jeunesse,

À l'argile animée par la flamme des cieux,

Et qui seront chantés demain par les poètes.

Car l'empire s'étend toujours plus loin à l'ouest,

Les quatre premiers actes sont déjà terminés,

Un cinquième finira le Drame avec le jour :

Le plus beau fruit du temps est toujours le dernier. revendications sociales de liberté, de responsabilité et de conscience individuelles se sont jointes très tôt à la vision esthético-éthico-sociale encouragée par Emerson. Emerson pensait ainsi favoriser une autonomie - et même un gai savoir - susceptible d'apporter une protection contre ce que Berkeley appelait déjà l'académisme, le pédantisme et autres perversions de ce genre. L'autonomie était pensée en conformité avec ce que Tocqueville appelle «la méthode philosophique des Américains». Elle dessinait son arrivée sur fond de récit de l'exode: un récit décisif en vertu duquel des personnes choisies ou élues seraient égales devant la loi nouvelle, tandis que d'autres, indésirables, périraient noyées dans les eaux profondes. Le chemin vers la terre promise est rarement accessible à tout le monde : il est réservé, généralement, à ceux qui savent se le frayer. Quand, un peu avant tout cela, Benjamin Franklin envisageait une prospérité nouvelle au sommet de la colline, il s'inspirait de la vision de John Bunyan, peinte en 1678 : une sorte de rêve chrétien des progrès du pèlerin, vers un État où l'on pourrait enfin se décharger de ses lourds bagages et se délivrer du fardeau du péché et de l'histoire. Franklin a écrit l'histoire de sa vie pour qu'elle serve d'exemple à la naissance d'une nation.

19 Si j'avais plus de temps, je vous parlerais du livre que je suis en train de terminer sur les récits de l'exode et d'émancipation dans l'histoire de la philosophie, dans les arts et dans l'opéra. L'essentiel de ce livre est un Passagen-Werk. J'y présente la vie qu'Arthur Danto a 
écrite pour lui-même comme un passage philosophique, où il s'agit de montrer comment une liberté nouvelle et parfaite pour les arts a pu être conquise en terre promise - et que cette terre a eu un rapport étroit avec Manhattan.

Alors que je terminais un texte pour un de ses livres, L'Abus de la beauté, publié en 2002, Danto m'a envoyé un mail. Il disait : «Je te joins le texte des conférences sur la beauté, et j'espère que tu l'apprécieras... Tu verras que j'ai emprunté la grande citation d'Adorno, qui, j'en suis sûr, n'aurait sans doute pas trouvé grand-chose de sympathique à tout ça. » La grande citation d'Adorno dont il est question est la déclaration sur laquelle s'ouvre sa Théorie de l'esthétique: «Il est devenu évident que tout ce qui concerne l'art, tant en luimême que dans sa relation au tout, ne va plus de soi, pas même son droit à l'existence ${ }^{10}$. " Danto ajoutait qu'il aurait aimé avoir écrit un livre commençant par cette phrase, et suggérait qu'il l'avait, en quelque sorte, déjà fait. Car la pensée exprimée ici était déjà la sienne; elle l'était plus ou moins depuis son article sur le monde de l'art de $1964^{11}$. Pour Danto comme pour Adorno, la perte de l'évidence marquait la fin d'un passage des Lumières qui avait mal tourné. Ils démontaient tous deux la logique historico-mondiale de l'absolutisme. Danto décrivait ce naufrage logique comme un désaffranchissement de la philosophie, laquelle avait transformé le monde de l'art en régime d'apartheid. Adorno décrivait le naufrage dans les termes sociaux et culturels de l'expulsion du mythe de la raison et du progrès, qui avaient été peu à peu instrumentalisés, administrés et rationnalisés. Tous deux auraient été d'accord avec ce que Toni Morrison a écrit dans son article "The Site of Memory ", à savoir que «l'Âge des Lumières" a aussi été celui du " racisme scientifique ${ }^{12}$ ». La science incluant bien sûr la philosophie, Wissenschaft. Mais le problème, c'est qu'elle avait inclus, consumé et remplacé tout le reste.

En 1778, Thomas Jefferson décrivait déjà en des termes forts ce qui était ou devait être évident pour tous. Ce qui l'avait été autrefois ne l'était plus. Ce qui l'était désormais avait l'approbation d'une voix unanime - à savoir qu'il ne se trouvait plus une seule voix pour ne pas vouloir dire aux colonies américaines qu'elles devaient et pouvaient se libérer du joug de l'Angleterre, incarné dans la figure du roi George III. Dans une évolution naturelle d'un récit de l'exode, le roi était assimilé à Pharaon, et la population des colonies aux Israélites souffrants. Les premières lignes de la Déclaration d'indépendance énonçaient donc ce qui était évident pour tous puisqu'aucune argumentation ni aucune guerre n'étaient plus nécessaires pour le démontrer. À savoir que «tous les hommes " étaient désormais égaux devant la loi de Dieu. Que les treize États seraient désormais « séparés » - indépendants - était le résultat naturel du " cours des événements humains », c'est-àdire de l'histoire. Ce qui donne, dans la traduction française de Jefferson lui-même :

Nous tenons pour évidentes pour elles-mêmes les vérités suivantes: tous les hommes sont créés égaux; ils sont doués par leur Créateur de certains droits inaliénables; parmi ces droits se trouvent la vie, la liberté et la recherche du bonheur.

Tout comme Danto parlerait plus tard du désaffranchissement de l'art par la philosophie, la Déclaration d'indépendance considérait «l'histoire du roi actuel de Grande-Bretagne » comme « l'histoire d'une série d'injustices et d'usurpations répétées qui toutes avaient pour but direct l'établissement d'une tyrannie absolue... » Tous ce que les citoyens avaient à faire était de faire respecter leurs droits en créant « un monde candide » et en dressant une liste des «Faits » de ce qu'« Il » - le roi - avait injustement refusé, interdit, dissous et empêché. Un historien au moins s'est intéressé non pas seulement à ce qui était déclaré dans le texte, mais aussi à la force performative de la Déclaration. L'inévitabilité, la nécessité, l'évidence, le sens commun de ses « vérités » s'appuient sur une performance 
par laquelle, comme devant un tribunal, les crimes du roi - ou du « Il» maintes fois répété - sont énumérés un à un avec insistance. Cela produit une sorte de poésie en $A B A$, une déclamation répétitive.

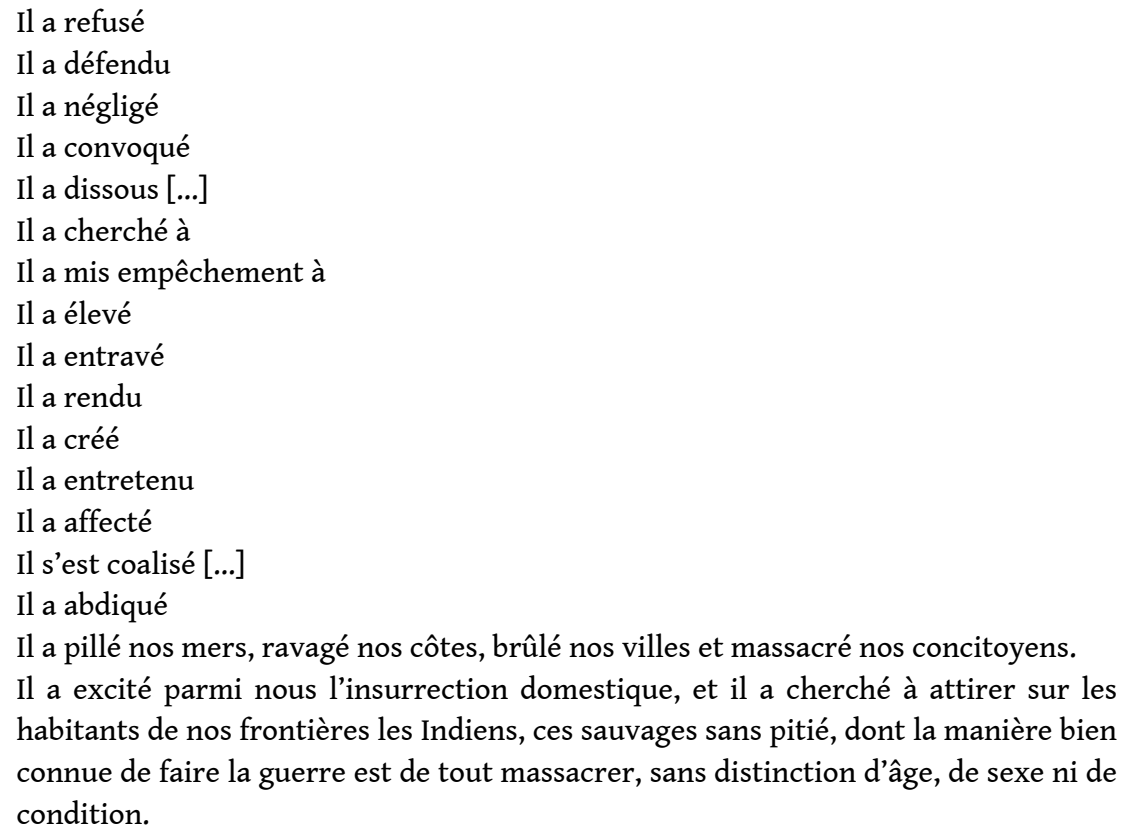

La justice de la Déclaration nécessitait la renaissance de la nation. Au-delà, philosophiquement, le texte s'appuyait solidement sur le Second traité du gouvernement de Locke, où avait été depuis longtemps donné à un autre peuple, en Angleterre déjà, le droit de renverser la tyrannie et de chercher la tolérance et la liberté.

Bien sûr c'est la violence du renversement qui a toujours été en question dans le discours de l'expansionnisme et de la révolution. Car s'il y a bien quelque chose d'évident à propos des Lumières, c'est que ce renversement violent peut transformer les vainqueurs en nouveaux tyrans. C'est cela qui a conduit beaucoup d'observateurs à interroger l'idée même d'une téléologie des Lumières et à s'attaquer à l'idée qu'elle était un passage qui légitimait la force brute, entre des commencements coupables et une fin divine. J'ai longtemps pensé qu'avec leur titre, Horkheimer et Adorno voulaient moins décrire que démonter la dialectique des Lumières, auquel cas, malgré ce titre, on devrait plutôt parler de « dialectique négative des Lumières ».

Prenons les conférences sur les Lumières qui ont été données par le philosophe américain Hilary Putnam à Amsterdam puis publiées en $2001^{13}$. Il juxtapose "trois âges des Lumières ", et si ses lectures vont de Platon à Rorty, rien ne montre qu'il ait jamais fait craquer la reliure du livre d'Horkheimer et Adorno. Une curieuse résistance empêche certains de lire Adorno - c'est un sujet à part entière. (Quand Danto m'a écrit pour me dire qu'il citait la première phrase de la Théorie esthétique, je lui ai recommandé de ne pas arrêter là sa lecture du livre. Il m'a dit qu'il ne jugeait pas cela nécessaire.)

Les trois âges des Lumières de Putnam commencent avec le dialogue de Platon intitulé Euthyphron. Comme Danto, il considère les Lumières comme un projet presque exclusivement réservé aux philosophes. Un philosophe éclairé, dans le style de Socrate, poursuit la justice et un mode critique de pensée. Ensemble, et pas séparément, les deux recherches permettent au philosophe de remettre en question l'autorité de la sagesse conventionnelle, du sens commun et des structures de l'évidence, d'une part, et l'autorité 
de la révélation, d'autre part. Quand la pensée critique oublie de rechercher la justice, son caractère réflexif et indépendant prend souvent fin après avoir pris fait et cause pour une nouvelle tyrannie ou pour un nouveau fanatisme. Les deuxièmes Lumières coïncident avec la nouvelle science ou avec le début de la philosophie moderne, c'est-à-dire Kant. Elles offrent une transcendance réflexive et une position de recul qui permettent d'examiner de façon critique les thèses, disons, de la loi naturelle et du contrat social. Elle se trompe généralement quand, dans la production de la théorie, la dispute verbale se confond avec les désaccords de fond, si bien que ceux-ci ne sont plus examinés. Les troisièmes Lumières, les seules qui fonctionnent si l'on en croit Putnam, viennent du pragmatisme américain, assaisonné d'une touche de Wittgenstein. Putnam s'inspire de ce que Dewey écrit sur «le criticisme du criticisme ", une formule qui, d'après les propres termes de la théorie critique, devrait plutôt être rédigée ainsi : « la critique des schémas établis du criticisme ». Les Lumières pragmatistes recommandent une démocratie délibérative qui ne prenne pas le bien commun au pied de la lettre, et qui ne soit pas fondée sur les sentiments d'empathie, de pitié ou de sympathie. Tout y est soumis à un examen permanent pour empêcher la rigidification des croyances et des sentiments. L'examen permanent, à la William James, exige d'introduire de nouveaux «conceptsobservations " pour répondre philosophiquement aux problèmes qui se posent réellement aux individus et aux sociétés. Putnam approuve Dewey quand celui-ci met l'accent sur l'éducation, sur l'expertise et sur les intérêts moraux transformateurs qui « laissent entrer de l'air frais ». Ces Lumières reconstruisent la démocratie, la raison et le progrès dans la perspective non de la certitude, mais de la faillibilité.

Putnam défend les Lumières pragmatistes en prenant position contre ce qu'il qualifie de gesticulation postmoderne, où les penseurs plongent trop rapidement dans l'océan après s'être débarrassés de leurs instruments de navigation. Il emprunte à Habermas l'idée d'un processus d'apprentissage permanent qui prend au sérieux la possibilité du progrès sans tenir pour acquise la certitude d'un progrès qui aurait déjà été réalisé. Il pense que le progrès en tant qu'idée ou idéal a été profondément malmené par des penseurs comme Foucault et Derrida, ou Bernard Williams et Rorty, qui estimaient que le progrès, et donc la philosophie, n'était qu'un jeu de pouvoir ou relevait d'une administration bureaucratique. S'il approuve l'archéologie quand elle permet de critiquer l'abus des concepts, Putnam rejette l'idée que la vérité ne serait qu'une question d'interprétation ou d'histoire. Quand Williams demande comment "cette» histoire est devenue «notre histoire ", Putnam acquiesce à nouveau, tout en rejetant la conclusion que le philosophe ne pourrait plus rien faire. Être un pragmatiste, c'est reconnaître qu'il est de son devoir de formuler des alternatives à ce que nous vivons aujourd'hui, sans tomber pour autant dans une certitude ou un absolutisme doctrinaires, et sans croire que l'alternative proposée soit la seule solution. Mais le pragmatiste ne doit pas non plus présenter ces solutions comme s'il n'était qu'un relativiste. Pour le pragmatiste éclairé, la vérité est entre les deux extrêmes de l'absolutisme et du relativisme, de la révélation et du nihilisme, et c'est le seul Lebensraum, me semble-t-il, qui ait vraiment fait des progrès éclairés.

Il est évident que Putnam a mal lu les prétendus "postmodernistes », ou du moins qu'il ne comprend pas ou n'accepte pas les raisons stratégiques et radicales des positions qu'ils ont prises en tant que philosophes contre la tyrannie du dogme philosophique et l'erreur sociale. Il est évident aussi qu'il vide les Lumières de l'essentiel de leur substance sociale et esthétique afin de pouvoir définir le bon chemin en avant pour les seuls philosophes. 
Enfin, son analyse des trois âges des Lumières diffère de la vision dialectique d'Horkheimer et Adorno. Car bien que ces derniers remontent à l'Antiquité et au début de la science moderne, ils ne font pas le compte des Lumières, eins, zwei, drei, mais retracent une trajectoire ayant une histoire longue et difficile. Ils se demandent moins combien il y a eu de Lumières, mais comment il a pu y en avoir à tel ou tel moment. Ce qui n'empêche pas qu'ils s'inspirent eux aussi du courant pragmatiste quand ils affirment que, vu la perte ou le manque de Lumières, quantitativement et qualitativement, il faudrait, comme chez Dewey, œuvrer à ce qu'il y en ait plus et qu'elles éclairent mieux.

Mon prochain mouvement porte sur les États-Unis entre les années 1930 et les années 1970, les décennies que l'historien Mark Greif a récemment rangées sous la rubrique de la pensée et de la fiction dans The Age of the Crisis of Man ${ }^{14}$. Ce livre est des plus pertinents pour aborder le discours sur la crise ou sur la catastrophe qui s'est emparé des universités et de la vie intellectuelle aux États-Unis. Il retrace les luttes entre penseurs, en particulier à Columbia, à Chicago et à la New School, au sujet des diverses façons de penser la crise, avec ou sans solution proposée. Greif commente la myriade de livres qui se demandent ce qu'est l'Homme, ou si l'Homme est la mesure, ou si l'homme moderne est obsolète ou en suspens, ou s'il est devenu trop difficile de trouver un homme bon. Le mot " homme » a bien sûr été contesté sans fin en raison de son extension vers le surhomme et le soushomme, ou à cause de l'homme de sexe masculin qui ne réussit pas à inclure les femmes, ou de l'homme blanc qui oublie d'inclure l'homme de couleur. Greif examine aussi la question de savoir si l'humanité, sous la rubrique des Lumières, du Destin, de l'Histoire ou du Léviathan, peut ou devrait être ressuscitée, renouvelée, reconstruite et revitalisée, et, si c'est le cas, par quels moyens de politique sociale, par quels outils, par quelle littérature, par quelle éducation.

En lisant la Dialectique des Lumières d'Horkheimer et Adorno, Greif compare ce livre à la Condition de l'Homme de Lewis Mumford ${ }^{15}$. Le premier a été publié dans une édition limitée de 500 exemplaires, en allemand, raison pour laquelle son " pessimisme extrême " n'a été accessible qu'à la communauté d'exilés germanophones aux États-Unis. Le second, publié en 1944 à New York chez Harcourt, était très américain, c'est-à-dire bien moins pessimiste, et il a été largement commenté lors de sa publication. Pourtant, Greif arrive à la conclusion qu'il est parfois impossible de dire à quel livre appartiennent les points de vue qu'ils défendent :

L'année 1944 a été extraordinaire pour le discours sur l'homme ; elle mêlait en effet la certitude absolue que les Alliés allaient gagner et la découverte pessimiste que les choses ne reviendraient jamais à la normale quand la victoire serait acquise... Pourtant, les deux livres ont en commun une thèse qui aurait été incompréhensible à toute autre époque : à savoir que le progrès, tel qu'il est attesté par l'histoire universelle du développement humain, pourrait contenir en lui une domination anti-progressiste risquant de mettre fin à l'émancipation de l'humanité16.

Après une comparaison de quelques phrases tirées des deux livres, Greif affirme que la question de 1944 était l'interrogation, projetée, de savoir si le fait de gagner la guerre contribuerait au progrès des Lumières. Et que les deux livres exprimaient la crainte que l'appareil industriel de la guerre mondiale fasse de la victoire sur «toute la terre éclairée " (pour la seconde fois) une victoire à la Pyrrhus, "rayonnante de calamités triomphales ». Greif exagère sans doute la similitude entre les deux thèses, mais sa lecture n'en est pas moins fort utile pour situer Horkheimer et Adorno dans ce débat américain. 
reif s'intéresse en particulier à l'année 1944, quand la victoire n'était pas aussi certaine qu'espérée. Pour mon dernier mouvement, j'aimerais rester à cette année. Il y a quelques mois, j'ai présenté un concert, à Paris, qui était annoncé dans les documents publicitaires comme « les États-Unis de la musique » (United States of Music). Le concert proposait deux nouvelles œuvres contemporaines de jeunes compositeurs américains, autour d' Appalachian Spring, d'Aaron Copland, qui date justement de 1944. Je me suis longtemps attardée sur le titre de ce programme. J'ai ouvert le site web du concert et j'ai découvert que le titre était censé signifier, en manière de slogan, une musique « made in USA », et même, plus précisément, "made in New York ». Cette provocation en forme de slogan était-elle bien utile? Je me suis demandée si, d'après la promesse de cette appellation commerciale, nous étions censés écouter ces œuvres en ayant la garantie qu'elles soient authentiquement américaines. Ou peut-être comme les seules représentantes de ce qui avait été produit et de ce qui était aujourd'hui produit aux États-Unis? Ou peut-être ce slogan était-il destiné à des oreilles françaises, à un public parisien qui s'apprêtait à recevoir quelque chose venant des États-Unis, quelque chose qui, compte tenu du climat politique actuel, pouvait ne pas être refusé a priori? Avait-on dit au public que ce qu'ils allaient recevoir était l'Amérique dans ce qu'elle avait de meilleur et non les États-Unis dans ce qu'ils ont de pire ? Une Amérique qui a autrefois accepté de la France une « Statue de la Liberté » pour incarner dans la pierre les libertés représentées par l'« Amérique ». Ou le concert devait-il être interprété comme un commentaire, depuis l'étranger, sur l'état non pas des États-Unis, mais de la France, où les libertés civiles sont aujourd'hui menacées?

Mon propos a principalement consisté à me demander comment, en prenant au sérieux des slogans et des publicités, il était possible de faire comprendre les différences possibles, mais problématiques, entre la musique américaine et une musique produite aux États-Unis. Ce que j'interrogeais, ce n'était donc pas le concert présenté simplement comme un concert de musique américaine. Peut-être voulais-je dire que, bien qu'elles viennent des États-Unis, les œuvres n'étaient pas authentiquement américaines, vu les origines européennes incontestables de cette musique. Et même si cette musique européenne était produite aux États-Unis depuis au moins deux siècles, elle n'avait jamais été jugée véritablement américaine, comme le sont le jazz, le blues, ou les musiques dites folk ou pop. Si cette idée a quelque substance, alors on pourrait dire que les œuvres de ce concert vivaient aux États-Unis comme des résidents étrangers - avec une carte verte qui pouvait leur être retirée à tout moment. D'un autre côté, penser la musique européenne comme n'ayant aux États-Unis qu'un statut de résident étranger, c'était alimenter la pire vision des États-Unis, celle d'un pays où l'on sépare ceux qui en font vraiment partie et ceux qui ne le seront toujours qu'à moitié. Il serait bien évidemment préférable de les voir comme un pays ouvert aux diverses communautés, où l'on ne met pas sur quelquesunes - et même sur la plupart - l'étiquette d'étrangers permanents.

Pour illustrer mon propos, j'ai raconté l'histoire de l'œuvre de Copland de 1944, Apalachian Spring. Elle est parfois connue comme une suite pour treize instruments et parfois comme une danse. Copland avait intitulé sa musique Ballet pour Martha, Martha Graham. Et Graham avait intitulé son ballet Appalachian Spring. Les musicologues ont décrit la pièce comme une œuvre hybride, où l'unité de la musique et de la danse découle de l'exécution et non pas de la volonté de l'auteur. Bien que Graham et Copland aient communiqué, ils ont voulu éviter que la danse soit soumise à la musique, et réciproquement. Ils ont donc travaillé chacun de leur côté, dans le but que l'unité naisse 
sur la scène. Ce fait $a$, en lui-même, des équivalents politiques et pragmatistes intéressants.

En 1943, la mécène Elizabeth Sprague Coolidge a commandé à Graham une danse pour un festival à la Bibliothèque du Congrès. Graham a contacté Copland pour créer une œuvre " américaine ». Envisageant plusieurs approches, ils se sont décidés pour le thème de l'optimisme américain, exprimé par un peuple de pionniers à la recherche de racines sur une terre nouvelle. Derrière la thématique explicite de l'œuvre, ils ont cependant suggéré divers messages et significations relatifs à la guerre, au fascisme et au nazisme ; à la lutte pour les droits et libertés civiques sur fond de guerre de Sécession; et au sentiment d'appartenance et de non-appartenance des pionniers, quand ils se frayaient un chemin dans un assemblage à peine uni d'États. Représenter un peuple de pionnier par la danse et par le chant, revenait pour eux à interroger les idées alors dominantes de front populaire de l'extrême gauche et plus encore de l'extrême droite.

Graham a emprunté son titre à un poème de Hart Crane intitulé « The Dance » (la danse) :

o Appalachian Spring! I gained the ledge;

Steep, inaccessible smile that eastward bends

And northward reaches in that violet wedge

of Adirondacks!-wisped of azure wands ${ }^{17}$,

Beaucoup ont vu dans Appalachian Spring une "icône américaine », représentant la liberté, l'individualisme et, après Stravinsky, les rites annuels du renouveau. Un critique dit de l'œuvre qu'elle exprime un « sens éternel de l'opportunité et de la promesse, tout en synthétisant un langage musical qui, comme le plus accompli de nos efforts nationaux, transforme la myriade de fils formant notre communauté collective en ensemble intégré merveilleusement beau ${ }^{18}$ ». Ce «sens éternel» est un sens utopique, un geste de transformation qui n'est pas autorisé à mourir.

Un lecteur plus critique, Mark Franko, décrit l'œuvre comme un palimpseste complexe, mais aussi comme un exercice de refoulement et d'autocensure, compte tenu du fait que les spécificités et les particularités locales des personnages et du récit sont abstraites et vidées de leur contenu ${ }^{19}$. Les personnages et les actions y deviennent typologiques : ils condensent des caractères sociaux pour créer un espace d'indétermination et des signifiés adaptables, un imaginaire d'ambiguïté saine et stratégique. Cette idée est conforme à l'idéal d' "Amérique » : un espace rêvé, un imaginaire politique dans lequel les blancs ne sont pas les seuls à pouvoir faire ce rêve - et atteindre la terre promise. Et pourtant, Franko observe que, dans les réceptions ultérieures d'Appalachian Spring, on a eu tendance à oublier complètement cet imaginaire politique originaire, et que c'est sans doute aux États-Unis que cet oubli a été le plus criant. Permettre à une œuvre de voyager dans le temps et dans l'espace est une manière, pour le public, d'oublier, au point parfois que l'œuvre ne représente plus du tout ce qu'elle était censée représenter au départ.

Copland affirmait travailler pour la cause des gens ordinaires. Il dit de sa contribution à la musique américaine qu'elle s'est inspirée d'idiomes folks comme les hymnes populaires, les chansons de cowboy, le jazz et la variété. Il a étudié auprès de Nadia Boulanger, sa professeure à Paris, mais aussi auprès de nombreux compositeurs qui ont cherché les racines nationales de la musique classique dans la musique populaire de leur pays natal : Liszt, Bartók, Dvořák, et bien d'autres. Malgré cet enracinement, beaucoup, dans les années 1920 surtout, ont vu dans leur musique un moyen de promouvoir un style international ou cosmopolite pour résister aux armées montantes qui se battaient au nom d'un nationalisme anti-Lumières. Un des airs les plus connus de Copland provient de la 
bataille de Waterloo, où un Écossais en jouait les notes sur sa cornemuse pour encourager son régiment à contraindre Napoléon à la retraite. Une autre référence d'Appalachian Spring est issue d'une chanson des Shakers (une congrégation protestante issue des Quakers) intitulée "Simple Gifts» (Simples dons). Par cette chanson, ce groupe remerciait Dieu de leur avoir permis, après avoir fui les persécutions en Angleterre, en 1774, d'avoir la liberté de chercher ailleurs une nouvelle patrie. La chanson «Simple Gifts » a été écrite en 1848, une année révolutionnaire capitale, quand l'idée de destinée manifeste faisait le tour du monde.

Je conclurai par un simple don, un extrait vidéo de la chorégraphie d'Appalachian Spring en 1944. L'homme qu'on voit danser est Merce Cunnigham. Et je me permets de revenir à l'idée de cette conférence en rappelant ceci : sept ans après la victoire alliée de 1945, Copland a été convoqué devant la Commission sur les activités antiaméricaines (House UnAmerican Activities Committee) pour y être interrogé sur sa fidélité aux États-Unis. La commission aurait dû être appelée "Commission des activités antiaméricaines" (Committee of UnAmerican Activities). Dans la dialectique des Lumières, changer une chose aussi modeste qu'une simple préposition, c'est changer toute son attitude par rapport au monde.

https://www.youtube.com/watch?v=E1o65tCZTWA

\section{NOTES}

1. ADORNO, Theodor Wiesengrund, et HORKHEIMER, Max, La Dialectique de la raison: fragments philosophiques, traduction française d'Éliane KAUFHOLZ, Paris, Gallimard, 1974.

2. GOEHR, Lydia, Elective Affinities: Musical Essays on the History of Æsthetic Theory, New York, Columbia University Press, 2008.

3. MERK, Frederick, Manifest Destiny and Mission in American History : A Reinterpretation, New York, A. A. Knopf, 1963, p. 3

4. Ibid., p. XVII.

5. Ibid.

6. Ibid.

7. SANTAYANA, George, The Life of Reason: or the Phases of Human Progress, New York, Scribner's sons, 1905, 5 vols.

8. RATZEL, Friedrich, Der Lebensraum : eine biogeographische Studie, Tubingen, Laupp, 1901.

9. PAINE, Thomas, " Appendix to the Third Edition ", Common Sense, 1776 ( $3^{\mathrm{e}}$ éd.).

10. ADORNO, Theodor W., Théorie esthétique, traduction française de Marc Jimenez, Paris, Klincksieck, 2011, p. 15.

11. DANTO, Arthur, "The Artworld", The Journal of Philosophy 61/19 (1964), p. 571-584; première traduction française in LORIES, Danielle, dir., Philosophie analytique et esthétique, Paris, Klincksieck, 1988, p. 183-198.

12. MORRISON, Toni, "The Site of Memory ", in ZINSSER, William, dir., Inventing the Truth: The Art and Craft of Memoir, New York, Houghton Mifflin, 1995 (2e éd.), p. 83-102.

13. PUTNAM, Hilary, Enlightenment and Pragmatism, Amsterdam, Van Gorcum, 2001. 
14. GREIF, Mark, The Age of the Crisis of Man: Thought and Fiction in America, 1933-1973, Princeton University Press, 2015.

15. MUMFORD, Lewis, The Condition of Man, New York, Harcourt, 1944.

16. GREIF, op. cit., p. 58.

17. Ô Printemps des Appalaches ! J'ai gagné le bord ; Sourire abrupt, inaccessible, qui s'incurve vers l'est Et touche côté nord cette encoignure mauve Des Adirondacks! - méchée de tiges azur.

18. MOUGHALIAN, Sato, "Aaron Copland's Appalachian Spring and the traditional music that inspired it ", http://perspectivesensemble.com/blog/perspectives-ensemble-notes/aaroncoplands-appalachian-spring-and-the-traditional-music-that-inspired-it (consulté le 28 mars 2017).

19. FRANKO, Mark, Martha Graham in Love and War: the Life in the Work, New York, Oxford University Press, 2012.

\section{INDEX}

Mots-clés : philosophie, musique, politique, États-Unis, Lumières

Keywords : philosophy, music, politics, United-States, Lumières

\section{AUTEUR}

\section{LYDIA GOEHR}

Lydia Goehr est professeure de philosophie à l'université Columbia de New York. Reconnue internationalement pour ses travaux en théorie esthétique, et en particulier sur les relations entre musique et politique, elle est notamment l'auteure de The Imaginary Museum of Musical Works : An Essay in the Philosophy of Music (Oxford University Press, 1992), The Quest for Voice: Music, Politics, and the Limits of Philosophy (Oxford University Press, 1998 ; traduction française sous le titre Politique de l'autonomie musicale : essais philosophiques, La rue musicale, 2016) et Elective Affinities: Musical Essays on the History of Æsthetic Theory (Columbia University Press, 2008). 